\title{
FREE AND DIRECT OBJECTS
}

\author{
BY Z. SEMADENI ${ }^{1}$
}

Communicated by Edwin Hewitt, July 18, 1962

1. General considerations. Let $B$ be a bicategory; ${ }^{2}$ the following terms are supposed to be familiar to the reader: object, morphism (=map of the class in the question), equivalence (=isomorphism) injection, surjection (=projection in the sense of $[13 ; 9])$. A morphism $\alpha: A \rightarrow B$ is called a retraction (and $B$ is called a retract of $A$ ) if there exists a cross-section $\beta: B \rightarrow A$ i.e., a morphism such that $\alpha \beta$ is the identity $\epsilon_{B}: B \rightarrow B$. If this is the case, $\alpha$ must be a surjection and $\beta$ must be an injection. $\operatorname{Map}(A, B)$ will denote the set of all morphisms $\alpha: A \rightarrow B$.

An object $S$ will be called a singleton if $\operatorname{Map}(S, A)$ is not void and $\operatorname{Map}(A, S)$ consists of exactly one morphism for every object $A$; dually $S$ is a cosingleton if $\operatorname{Map}(A, S) \neq \varnothing$ and $\operatorname{Map}(S, A)$ consists of exactly one morphism for every $A$. All singletons and cosingletons are equivalent (if they exist). $S$ is a singleton and a cosingleton simultaneously if and only if it is a null object. An example of a singleton which is not a null object is a one-point space in the category of topological spaces.

$\left\{A_{t}\right\}_{t \in T}$ being a set of objects, $\Sigma A_{t}$ and $\Pi A_{t}$ will denote the free and direct join of it (cf. $[12, \S 12]$ ) with monomorphisms $\sigma_{t}: A_{t} \rightarrow \Sigma A_{u}$ and epimorphisms $\pi_{t}: \Pi A_{u} \rightarrow A_{t}$, respectively.

PROPOSITION 1. If \& has a singleton or a cosingleton, then the monomorphisms $\sigma_{t}: A_{t} \rightarrow \Sigma A_{u}$ are injections admitting retractions $\pi_{t}: \Sigma A_{u} \rightarrow A_{t}$ and, dually, the epimorphisms $\pi_{t}: \Pi A_{u} \rightarrow A_{t}$ are surjections admitting cross-sections $\sigma_{t}: A_{t} \rightarrow \Pi A_{u}$.

According to the standard definition an object $P$ is projective if for every surjection $\alpha: A \rightarrow B$ and every $\beta: P \rightarrow B$ there exists $\gamma: P \rightarrow A$ such that $\alpha \gamma=\beta$, and $I$ is injective if for every injection $\alpha: B \rightarrow A$ and every $\beta: B \rightarrow I$ there exists $\gamma: A \rightarrow I$ with $\gamma \alpha=\beta$.

PRoposition 2. The retracts and free joins of projective objects are projective; the retracts and direct joins of injective objects are injective.

An object $M$ with be called a coseparator if for any two objects $A$ and $B$ and for any morphisms $\alpha: A \rightarrow B$ and $\beta: A \rightarrow B$, the condition $\alpha \gamma=\beta \gamma$ for all $\gamma \in \operatorname{Map}(M, A)$ implies $\alpha=\beta$. Let us notice that any

${ }^{1}$ Research supported partially by the National Science Foundation.

2 We assume Isbell's system of axioms, cf. [9], also [5; $7 ; 12 ; 13]$. 
coseparator is a generator in the sense of [7].

An object $F$ will be called a basic free object (abbreviation: b.f.o.) if the following conditions are satisfied: (1) $F$ is a coseparator. (2) If a coseparator $A$ is a retract of $F$, then $A$ is equivalent to $F$. (3) If $B$ is any coseparator, then there exists a retraction $\alpha: B \rightarrow F$. A b.f.o. $F$ will be called strict if the following condition holds: If $\alpha: B \rightarrow A$ is an injection and the conjugate map $\alpha^{\prime}: \operatorname{Map}(F, B) \rightarrow \operatorname{Map}(F, A)$ is onto, then $\alpha$ is an equivalence.

$F$ is unique up to equivalence (if it exists). An object $P$ will be called free if it is a free join of a set of copies of $F$. Dually we define: a separator, a basic direct object $D$ (abbreviation: b.d.o.), a direct object, a strict b.d.o.

THEOREM. Suppose that $B$ admits a singleton or a cosingleton and free [direct] objects and that b.f.o. is strict and projective [b.d.o. is strict and injective]. Then

(i) Every free [direct] object is projective [injective].

(ii) Every object is an image of a free object [a subobject of a direct object] (i.e., for each $A$ there exists a surjection $\alpha: \Sigma(F)_{t} \rightarrow A$ [an injection $\left.\left.\alpha: A \rightarrow \amalg(D)_{t}\right]\right)$.

(iii) An object is projective [injective] if and only if it is a retract of a free [direct] object.

(iv) $A n$ object $A$ is projective [injective] if and only if for every object $B$ every surjection $\alpha: B \rightarrow A$ has a cross-section [every injection $\alpha: A \rightarrow B$ has a retraction]. In other words, $A$ is projective [injective] if and only if it is an absolute quotient retract [absolute subretract].

2. Examples. 1. If $B$ is the bicategory of abelian groups and homomorphisms, then the infinite cyclic group $Z$ is a b.f.o. and $Q / Z$ is a b.d.o. where $Q$ denotes the group of rationals. Free objects are just free abelian groups, every projective is free and injective objects are just the divisible groups (cf. $[11, \S 12 ; 13]$ ).

2. If $B$ is the bicategory of all groups and homomorphisms then $Z$ is a b.f.o. Free objects are the same as free groups and projective objects are the same as free (Nielsen-Schreier theorem, cf. [11,\$35]). The only injective object is the null object [3, Theorem 2].

3 . If $B$ is the bicategory of Boolean algebras and Boolean homomorphisms, then the two-element algebra $(0,1)$ is a cosingleton and a b.d.o. while a four-element algebra $\left(0,1, A, A^{\prime}\right)$ is a b.f.o. Free objects are those which have a free system of generators and an algebra is a direct object if and only if it is the field of all subsets of a set. Injective objects are just the complete algebras (cf. $[21 ; 8]$ ).

4. If $B$ is the bicategory of left $\Lambda$-modules (cf. [4]), then $\Lambda$ is a 
projective coseparator and $\operatorname{Hom}_{Z}(\Lambda, Q / Z)$ is an injective separator.

5. If $B$ is the bicategory of compact Hausdorff spaces and continuous maps, then a one-point space is a singleton and a b.f.o. simultaneously and a closed interval is a b.d.o. Free joins are $\beta\left(U S_{\alpha}\right)$ (where $U S_{\alpha}$ is the disjoint union) and direct joins are Cartesian products, whence free objects are just $\beta\left(N_{\alpha}\right)$ with $N_{\alpha}$ discrete and direct objects are the Tichonov cubes. Projective objects are just the extremally disconnected ones (cf. $[6 ; 16]$ ) and injective objects are absolute retracts.

6 . If $B$ is the bicategory of normed linear spaces and linear operators with $\|T\| \leqq 1$ (injections being isometries into), then the real line is both b.f.o. and b.d.o. Free joins are $l_{1}$-direct sums i.e., spaces of functions $t \rightarrow x_{t}$ with $x_{t} \in X_{t}$ and $\left\|\left\{x_{t}\right\}\right\|=\sum\left\|x_{t}\right\|<\infty$, and direct joins are $m$-direct sums i.e., spaces of bounded functions $t \rightarrow x_{t}$ with $x_{t} \in X_{t}$ and $\left\|\left\{x_{t}\right\}\right\|=\sup \left\|x_{t}\right\|$. Thus, free objects are the spaces $l_{1}\left(N_{\alpha}\right)$ and direct objects are the spaces $m\left(N_{\alpha}\right)$. Projective objects are the same as free and injective ones are those with the binary intersection property i.e., those equivalent to spaces $C(S)$ with $S$ extremally disconnected.

The bicategory of normed linear spaces and all linear (continuous) operators (injections being bicontinuous) does not admit infinite free or direct joins. The spaces $l_{1}\left(N_{\alpha}\right)$ and $m\left(N_{\alpha}\right)$ are projective and injective, respectively. No characterization of projective and injective objects is known. For references see $[2 ; 10 ; 14 ; 15 ; 19]$.

7. The bicategory of normal two-norm spaces and $\gamma-\gamma$-linear operators (cf. [1]) with $\|T\|=\sup \{\|T x\|:\|x\| \leqq 1\} \leqq 1$ admits free and direct joins of countable sets of objects (defined as $l_{1}$-products and $m$-products). No nonzero object is injective (cf. [17]). The real line is injective in the category of $\boldsymbol{\gamma}$-reflexive spaces and all $\boldsymbol{\gamma}$ - $\boldsymbol{\gamma}$-linear maps (cf. [18]) but it is not injective for $\gamma$ - $\gamma$-linear maps with $\|T\| \leqq 1$ because the number $\epsilon$ in Theorem 6 of [18] is indispensable.

The proofs and details will be published in [20].

\section{REFERENCES}

1. A. Alexiewicz and $Z$. Semadeni, The two-norm spaces and their conjugate spaces, Studia Math. 18 (1959), 275-293.

2. D. Amir, Continuous functions spaces with the bounded extension property, Bull. Res. Council Israel 10F (1962), 133-138.

3. R. Baer, Absolute retracts in group theory, Bull. Amer. Math. Soc. 52 (1946), 501-506.

4. H. Cartan and S. Eilenberg, Homological algebra, Princeton Univ. Press, Princeton, N. J., 1956.

5. S. Eilenberg and S. MacLane, General theory of natural equivalences, Trans. Amer. Math. Soc. 58 (1945), 231-294. 
6. A. M. Gleason, Projective topological spaces, Illinois J. Math. 2 (1958), 482-489.

7. A. Grothendieck, Sur quelques points d'algèbre homologique, Tôhoku Math. J. 9 (1957), 119-121.

8. P. R. Halmos, Injective and projective Boolean algebras, Proc. Sympos. Pure Math. Vol. 2, pp. 114-122, Amer. Math. Soc., Providence, R. I., 1961.

9. J. R. Isbell, Some remarks concerning categories and subspaces, Canad. J. Math. 9 (1957), 563-577.

10. J. R. Isbell and Z. Semadeni, Projection constants and spaces of continuous functions, Trans. Amer. Math. Soc. (to appear).

11. A. G. Kurosh, The theory of groups, Vols. 1 and 2, Chelsea, New York, 1955 and 1956 .

12. A. G. Kurosh, A. H. Livshits and E. G. Shulgeifer, Foundations of the theory of categories, Russian Math. Surveys-translation from Uspehi Mat. Nauk (6), 15 (1960), 1-46.

13. S. MacLane, Duality for groups, Bull. Amer. Math. Soc. 56 (1950), 485-516.

14. L. Nachbin, Some problems in extending and lifting continuous linear transformations, Proc. Intern. Symp. on Linear Spaces, 1960, Jerusalem, 1961, 340-351.

15. A. Pelczynski, Projections in certain Banach spaces, Studia Math. 19 (1960), 205-228.

16. J. Rainwater, $A$ note on projective resolutions, Proc. Amer. Math. Soc. 10 (1959), 734-735.

17. Z. Semadeni, Embedding of two-norm spaces into the space of bounded continu. ous functions on a half-straight line, Bull. Acad. Polon. Sci. Sér. Sci. Math. Astr. Phys. 8 (1960), 421-426.

18. - Extension of linear functionals in two-norm spaces, ibid., 427-432.

19. - Isomorphic properties of Banach spaces of continuous functions, Proceedings of a Conference on Functional Analysis (Warsaw, 1960), (to appear).

20. ——, Projectivity, injectivity and duality, Rozprawy Mat. (to appear).

21. R. Sikorski, Boolean algebras, Ergebnisse Math. Heft 21, Berlin, 1960.

The Institute of Mathematics of the Polish Academy of Sciences and THE UNIVERSITY OF WASHINGTON 